\title{
Berufshaftpflichtversicherung für selbständige Ärzte
}

\section{Günstige, unkomplizierte Angebote für alle Fachrichtungen}

\section{Versicherungsnotstand in der Berufshaftpflicht für Ärzte?}

Wiederholt sind sicherlich auch Sie in den letzten Monaten auf diese Beurteilung gestossen. Seitens der FMH wurde eine Fachgruppe eingesetzt, welche Anfang 2006 zum klaren Schluss gekommen ist, dass das Angebot seitens der Versicherer ausreichend vorhanden ist.

\section{Unser Angebot}

Die FMH Insurance Services, Versicherungspartner Ihrer Standesorganisation, verfügen über attraktive Versicherungslösungen in sämtlichen Fachrichtungen mit Ausnahme der Schönheitschirurgie. Wir haben die Angebote unserer Versicherungspartner ausgiebig geprüft und mit führenden Gesellschaften teilweise exklusive Rahmenvereinbarungen getroffen. Profitieren Sie von unseren Spezialangeboten, welche Sie ausschliesslich über die FMH Insurance Services beziehen können. Nutzen Sie das ausgezeichnete PreisLeistungs-Verhältnis.

\section{Profitieren Sie jetzt und bestellen Sie Ihre persönliche Offerte}

Ihre Bedürfnisse stehen bei uns im Mittelpunkt. Mit unserem Namen garantieren wir Ihnen die Betreuung durch professionelle Berater mit langjähriger Erfahrung im Versicherungs- und Bankenbereich. Dank unserer Unabhängigkeit können wir Ihnen das für Sie am besten geeignetste Produkt anbieten.

\section{Antworttalon}

Vorname / Name

Adresse

PLZ / Ort

Geburtsdatum

Telefon Privat/Geschäft

Beste Zeit für einen Anruf

Facharzt für

Spezialitäten/Eingriffe/Behandlungen

Bestehende Haftpflichtversicherung

ja (Bitte eine Policenkopie beilegen)

nein

Ich wünsche eine persönliche Offerte.

Ich interessiere mich für folgende Produkte:
Pensionskasse BVG
Krankenkasse
O Säule $3 a$
Rechtsschutzversicherung
Finanz-/Steuerplanung
O Hausratversicherung 